\title{
Ultrafast valence intersubband hole relaxation in InGaN multiple-quantum-well laser diodes
}

\author{
Kian-Giap Gan ${ }^{\text {a) }}$ \\ Department of Electrical and Computer Engineering, University of California, Santa Barbara, \\ California 93106-9560 \\ Chi-Kuang Sun \\ Department of Electrical Engineering and Graduate Institute of Electro-Optical Engineering, \\ National Taiwan University, Taipei 10617, Taiwan, Republic of China \\ Steven P. DenBaars and John E. Bowers \\ Department of Electrical and Computer Engineering, University of California, Santa Barbara, \\ California 93106-9560
}

(Received 17 November 2003; accepted 14 April 2004; published online 19 May 2004)

\begin{abstract}
The ultrafast carrier dynamics in InGaN multiple-quantum-well (MQW) laser diodes were investigated using a time-resolved bias-lead monitoring technique. From the optical selection rules of TE and TM polarized light, one can selectively excite and probe different valence-subband-to-conduction-subband transitions in the MQW structure with different polarized pump and probe light. The subband structure of the MQW structure of the laser diode was calculated and is verified by electroluminescence measurement. Using this technique, ultrafast valence intersubband hole relaxation processes $(\tau<0.35 \mathrm{ps})$ were found to dominate the observed carrier dynamics. (C) 2004 American Institute of Physics. [DOI: 10.1063/1.1760211]
\end{abstract}

The group-III nitride semiconductor alloys AlN-GaN$\mathrm{InN}$ are recognized as an important material system for the optoelectronic devices in the spectral range from infrared to ultraviolet. GaN-InN based III-V nitride semiconductors are of interest in many commercial applications, such as light emitting diodes and laser diodes. ${ }^{1,2}$ The carrier dynamics, which are important for high speed device design, have recently been studied by femtosecond time-resolved pump- probe $^{3}$ or coherent spectroscopy ${ }^{4,5}$ with above-bandgap photons. However, various contributions such as electron-electron scattering, hole-hole scattering, electronhole scattering, electron-phonon interactions, and holephonon interactions, mix together and make it very difficult to extract the fundamental material parameter for one particular scattering process or single type of carrier. Sun et al. and Ye et al. have developed an infrared pump-ultraviolet probe technique to isolate electron and hole dynamics and used it to study the electron relaxation dynamics in $n$-type $\mathrm{GaN}$ thin films ${ }^{6,7}$ and hole dynamics in $p$-type GaN thin films. ${ }^{8}$ In this letter, we used a time-resolved bias-lead monitoring pump-probe technique ${ }^{9}$ that uses two UV pulses of equal amplitude with various polarization configurations (TE-TE, TM-TE, and TM-TM) to study the carrier dynamics in the InGaN multiple quantum well (MQW) laser diode. The TM polarization is the direction of electric field parallel to the $c$ axis (normal to the surface) and TE polarization is the direction of electric field perpendicular to the $c$ axis. From the optical selection rules of TE and TM polarized light, one can selectively excite and probe different valence subbands to conduction band transitions in the MQW structure with different polarized pump and probe light.

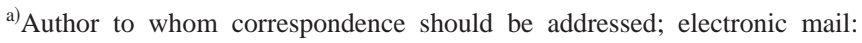
giap@ece.ucsb.edu
}

The sample under investigation was a commercial ridge waveguide MQW InGaN laser diode. The lasing wavelength of the laser diode is $403.5 \mathrm{~nm}$. The peak of TE polarized electroluminescence was $407.3 \mathrm{~nm}$ and the TM polarized electroluminescence was peaked at $400.7 \mathrm{~nm}$. The peak of the electroluminescence at TE and TM are separated by 6.6 $\mathrm{nm}(50 \mathrm{meV})$. This is consistent with the calculation that will be shown later.

In the wurtize crystalline structure, the selection rules for the optical momentum matrix elements for the transitions between the conduction band and the three valence bands can be derived from the symmetry properties of the zone center wave function. ${ }^{10,11}$ In the following, these acronyms will be used, C: conduction, HH: heavy hole, LH: light hole and $\mathrm{CH}$ : crystal-field splitoff hole. Let us first describe the band structure and the optical selection rule of bulk $\mathrm{In}_{0.15} \mathrm{Ga}_{0.85} \mathrm{~N}$. At the zone center $(k=0)$, the $\mathrm{HH}-\mathrm{C}$ transition will only occur when the polarization of the light is perpendicular to the $c$ axis, i.e., TE polarized. The $\mathrm{CH}-\mathrm{C}$ transition will favor the TM polarized light, i.e., the light polarized along the $c$ axis. For the $\mathrm{LH}$ band, $\mathrm{LH}-\mathrm{C}$ transition will mostly occur when the light is TE polarized. Away from the zone center, the $\mathrm{HH}-\mathrm{C}$ transition remains TE polarized while the $\mathrm{CH}-\mathrm{C}$ transition and the $\mathrm{LH}-\mathrm{C}$ transition switch polarization, i.e., $\mathrm{CH}-\mathrm{C}$ transition become TE polarized and LH-C become TM polarized. Because the hole energy of the $\mathrm{CH}$ band is larger than the hole energy of the HH band and LH band at the zone center, TM polarized light will excite holes with higher energy compared to the energy of the holes excited by the TE polarized light. The holes excited by TM polarized light will relax back to the top of the valence band and thus affect the absorption properties of TE polarized light, but not the other way around, i.e., TM will affect TE but TE will not affect TM. 

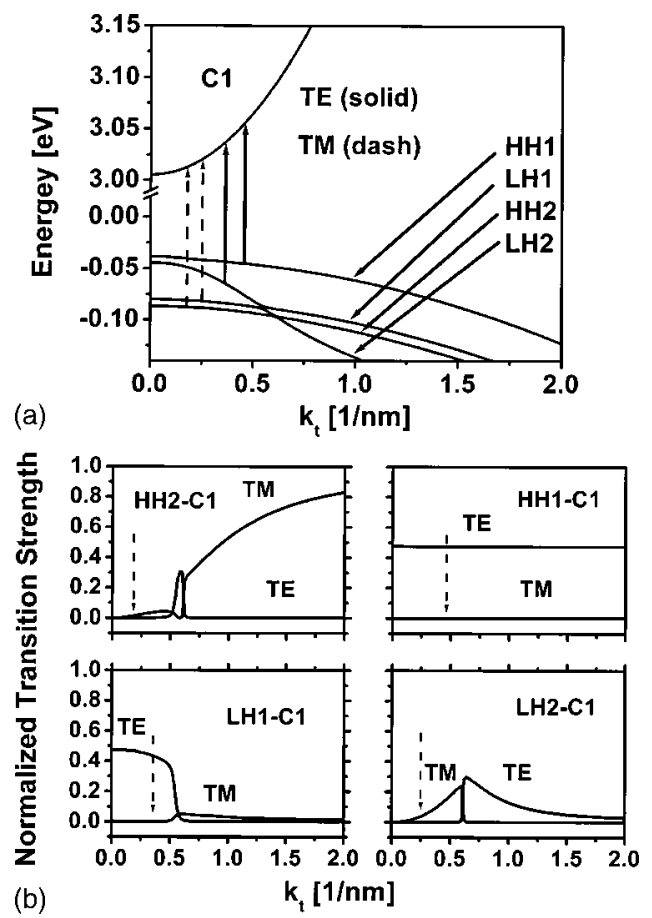

FIG. 1. (a) Calculated subband structure of the quantum well structure and (b) normalized transition strength for selected subband transition. TE polarized transition strength is normalized to $\left|\left\langle S\left|p_{x}\right| X\right\rangle\right|^{2}$ and TM polarized transition strength is normalized to $\left|\left\langle S\left|p_{z}\right| Z\right\rangle\right|^{2}$. The transitions corresponding to $400 \mathrm{~nm}$ light are indicated with vertical lines.

In the quantum well (QW) structure, the valence band turns into different valence subbands. Because of the valence band mixing effect, the optical selection rules are modified. We used the finite-difference method to solve the effectivemass equations ${ }^{12}$ for the QW structure. We took band structure parameters from Ref. 13 and a valence band offset of $33 \%$ was used. The band gap of $\mathrm{HH} 1-\mathrm{C} 1$ has been adjusted to match the peak of the measured TE polarized electroluminescence spectrum $(407.3 \mathrm{~nm})$. Figure 1(a) shows the dispersion curve of different conduction and valence subbands in the QW structure with $3 \mathrm{~nm} \quad \operatorname{In}_{0.15} \mathrm{Ga}_{0.85} \mathrm{~N}$ well and $\mathrm{In}_{0.02} \mathrm{Ga}_{0.98} \mathrm{~N}$ barrier. The solution of the effective mass equation was used to calculate the transition matrix element in order to find the optical transition strength for different valence subbands to conduction band transitions. The normalized optical transition strengths for the four lowest valence subband to the first conduction subband transitions are shown in Fig. 1(b). Note that the first significant TM polarized transition occurs at a higher energy compared with the TE polarized transition and the calculated energy separation $(\sim 50 \mathrm{meV})$ is consistent with the electroluminescence measurement. Since the TM polarized light will excite the higher energy hole compared to the TE polarized light, the same prediction for the bulk InGaN will also apply to InGaN QW, i.e., TM will affect TE but TE will not affect TM.

The schematic diagram of the time-resolved bias monitoring setup is shown in Fig. 2. The pump and probe beam are derived from the second-harmonic generation of a tunable 100 fs Ti:sapphire modelocked laser. Pump and probe beams are combined collinearly and directed to the laser diode under test. Both the pump and probe beams are mechanically chopped at frequencies of 1.7 and $2.0 \mathrm{kHz}$, respecDownloaded 16 Feb 2009 to 140.112 .113 .225 . Redistribution subje

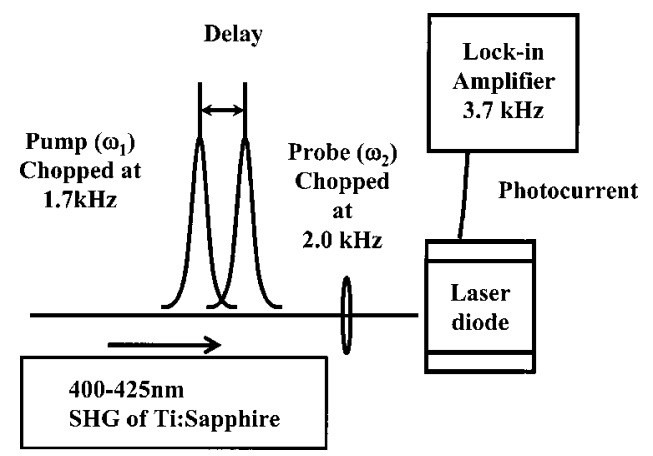

FIG. 2. Schematic diagram of experiment setup.

tively. The photocurrent collected from the laser diode was measured by a lock-in amplifier at the sum frequency of 3.7 $\mathrm{kHz}$ as a function of the delay between the pump and probe pulses. In order to avoid the interference signal between the pump and probe in the co-polarization configuration, the frequency of the probe beam was shifted by $40 \mathrm{MHz}$ with an acousto-optic frequency shifter.

At below-band-gap excitation (425 $\mathrm{nm})$, the timeresolved photocurrent response signal is enhanced when both pump and probe light overlapped in time. This positive instantaneous signal is attributed to two-photon absorption contributed with one pump photon and one probe photon and the width of this signal is $0.37 \mathrm{ps}$, limited by the autocorrelation width of the laser pulse.

When we tune the laser wavelength to be above the band gap of InGaN MQW, different behavior was observed for different pump-probe polarization configurations. Figure 3 shows examples traces taken at a wavelength of $400 \mathrm{~nm}$. As shown in Fig. 3(a), when both pump and probe are TE polarized, there is a negative instantaneous signal and a negative double-sided exponential decay signal. The negative instantaneous signal is attributed to a phase space filling effect with a fast initial relaxation faster than our system time resolution. This initial fast relaxation can be attributed to the carrier thermalization mainly due to carrier-carrier scattering. The slower negative exponential decay signal with a time constant $\left(t_{R}\right)$ of $2.2 \mathrm{ps}$ is attributed to the carrier energy relaxation where the carrier-phonon interaction will lead to a new equilibrium between the carriers and the lattice system. However, when both pump and probe are TM polarized, only a negative instantaneous signal can be observed. From Fig. 1(a), both HH1-C1 and LH1-C1 transitions can be excited at $400 \mathrm{~nm}$ with TE polarized light and the related electron dynamics may become relevant to the observed dynamics. ${ }^{14}$ However our observation reveals that the observed carrier dynamics are mainly contributed from the hole. The fact that the $2.2 \mathrm{ps}$ time constant process is missing in the TM-TM measurement and only exists in the TE-TE measurement suggests that the 2.2 ps process does not originate from the electron in the conduction band but from the hole in the valence band. The resolution-limited response in the TM-TM measurement suggests an extremely fast $(\tau$ $<0.35 \mathrm{ps}$ ) intersubband hole relaxation for the TM-generated hole in the LH2 and HH2 subbands into lower HH1 and LH1 subbands, which are only sensitive to the TE polarized light. In order to study this intersubband hole relaxation process, to AIP license or copyright; see http://apl.aip.org/apl/copyright.jsp 

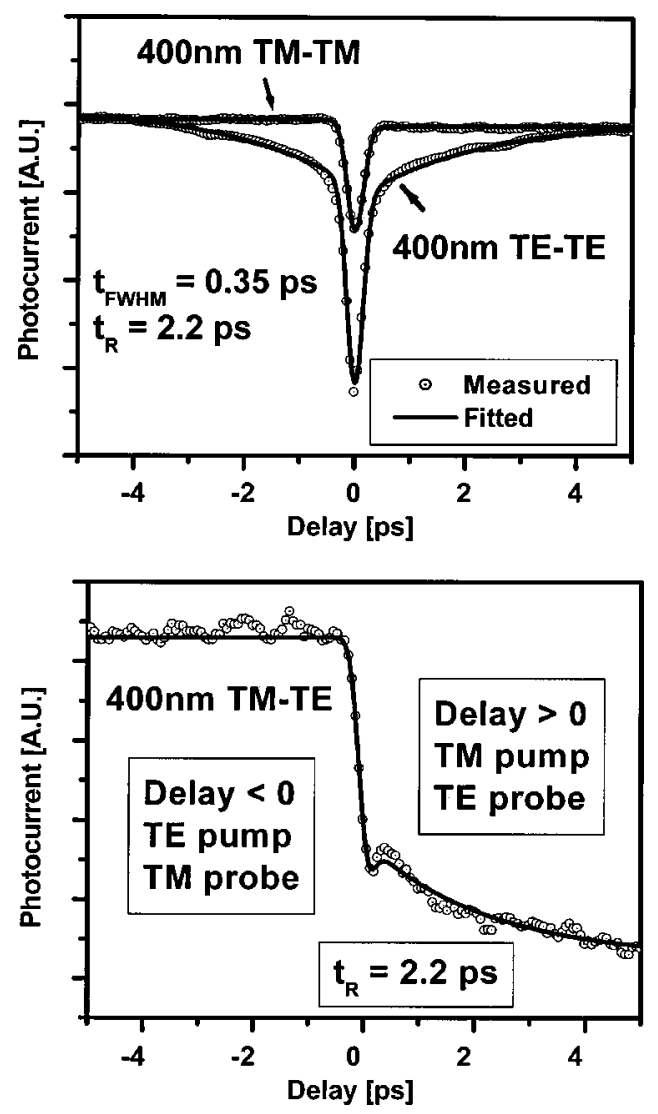

FIG. 3. Time-resolved photocurrent signal (arbitrary unit) with $400 \mathrm{~nm}$ excitation (carrier density $\sim 10^{17} \mathrm{~cm}^{-3}$ ): (a) TE-TE polarization and TM-TM polarization and (b) TM-TE polarization.

cross polarization measurement was performed and the result is shown in Fig. 3(b).

In the cross polarization configuration, positive delay means TM polarized light (pump) enters the laser diode before the TE polarized (probe) light and negative delay means TE polarized light (pump) enters the laser diode before TM polarized light (probe). At positive delay, there is fast initial decay followed by another positive single-sided exponential decay signal with the same time constant $\left(t_{R}=2.2 \mathrm{ps}\right)$ as the one observed in the TE-TE polarization configuration. The fast initial rise of the observed TE signal supports the previous suggestion that an extremely fast $(\tau<0.35 \mathrm{ps})$ intersubband hole relaxation for the TM-generated holes in LH2 and HH2 subbands relaxed into the HH1 and LH1 subbands. These LH2- and HH2-subband-transferred holes in the lower HH1 and LH1 subbands will then follow a similar thermalization process as the directly generated holes. It is interesting to note that at negative delay, the signal remains constant and the 2.2 ps process is not observed, further confirming that the 2.2 ps process is due to the generated hole and not due to the generated electron in the conduction band.

In conclusion, the femtosecond carrier dynamics in InGaN MQW laser diode were studied using a time-resolved bias-lead monitoring technique. Using the optical selection rules in the wurtize QW structure and various pump-probe polarization configurations, an ultrafast intersubband hole relaxation process $(\tau<0.35 \mathrm{ps})$ can be observed. We believe these ultrafast intersubband hole transitions will have a profound influence on the laser gain dynamics of $\mathrm{InGaN}$ laser diodes.

${ }^{1}$ S. Nakamura, M. Senoh, and T. Mukai, Appl. Phys. Lett. 62, 2390 (1993).

${ }^{2}$ S. Nakamura, M. Senoh, S. Nagahama, N. Iwasa, T. Yamada, T. Matsushita, H. Kiyoku, Y. Sugimoto, T. Kozaki, H. Umemoto, M. Sano, and K. Chocho, Appl. Phys. Lett. 72, 211 (1998).

${ }^{3}$ C.-K. Sun, F. Vallee, S. Keller, J. E. Bowers, and S. P. DenBaars, Appl. Phys. Lett. 70, 2004 (1997).

${ }^{4}$ S. Pau, J. Kuhl, F. Scholz, V. Haerle, M. A. Khan, and C. J. Sun, Phys. Rev. B 56, 12718 (1997)

${ }^{5}$ R. Zimmermann, A. Euteneuer, J. Mobius, D. Webber, and M. R. Hofmann, Phys. Rev. B 56, 12722 (1997).

${ }^{6}$ C.-K. Sun, Y.-L. Huang, S. Keller, U. K. Mishra, and S. P. DenBaars, Phys. Rev. B 59, 13535 (1999).

${ }^{7}$ H. Ye, G. W. Wicks, and P. M. Fauchet, Appl. Phys. Lett. 74, 711 (1999).

${ }^{8}$ H. Ye, G. W. Wicks, and P. M. Fauchet, Appl. Phys. Lett. 77, 1185 (1999).

${ }^{9}$ K. L. Hall, E. P. Ippen, and G. Eisenstein, Appl. Phys. Lett. 57, 129 (1990)

${ }^{10}$ G. L. Bir and G. E. Pikus, Symmetry and Strain-Induced Effects in Semiconductor (Wiley, New York, 1974).

${ }^{11}$ S. L. Chuang and C. S. Chang, Appl. Phys. Lett. 68, 1657 (1996).

${ }^{12}$ S. L. Chuang and C. S. Chang, Semicond. Sci. Technol. 12, 252 (1997).

${ }^{13}$ Y. C. Yeo, T. C. Chong, and M. F. Li, J. Appl. Phys. 83, 1429 (1998).

${ }^{14}$ A. M. T. Kim, S. Hunsche, T. Dekorsy, and H. Kurz, Appl. Phys. Lett. 68, 2956 (1996) 